\title{
A CONCEPT OF SEPARATED NUMERICAL FORMULATIONS FOR THE SOLUTION AND EVALUATION OF COMPLEX FIELD PROBLEMS
}

\author{
ANDRÉ BUCHAU \& MATTHIAS JÜTTNER \\ University of Stuttgart, Institute for Theory of Electrical Engineering, Stuttgart, Germany.
}

\begin{abstract}
Nowadays, a variety of numerical methods and numerical formulations exits to solve complex or coupled field problems in three dimensions. Most of them are generally applicable to nearly arbitrary kind of field problems. On the other hand, some highly optimized methods are available, which are predestined for the solution of a specific kind of problem. Especially in the case of weakly coupled multiphysics problems, a mixture of several numerical methods is very advantages to benefit from different properties of numerical methods for diverse physical sub-problems. A very promising approach for a flexible coordination of the related solution process is the application of software agents. Then, the results of one sub-problem are converted into boundary values or volume source distributions for another sub-problem and software agents choose solution methods independently for each subproblem. Furthermore, two main aspects have to be considered in applications of numerical methods. First, the solution of a boundary value problem should be computed efficiently and second, the solution is evaluated for visualization and interpretation of obtained results. In practice, it is difficult to choose a single appropriate method, which is well suited both for the solution of a problem and its evaluation, since the demands differ in both cases. Here, a concept is presented to apply various numerical methods successfully to the solution and evaluation of complex field problems. Attention is mainly turned on the integration of boundary element methods into the concept of mixed numerical formulations.
\end{abstract}

Keywords: boundary element methods, coupled problems, finite element methods, post-processing, software agent systems, visualization

\section{INTRODUCTION}

In the last years, the interest on the numerical solution of complex and coupled field problems has grown noticeably. Modern computers are equipped with relatively large main memory and modern central processing units (CPU) support the parallel computation of more than eight threads even for floating point arithmetic. Hence, large problems can be solved in acceptable time. Furthermore, very good implementations of general applicable numerical methods like the finite element method (FEM) or boundary element method (BEM) exist. Along with the increasing computation capability, the demand on numerical field simulations grows, too. Instead of stationary simulations, time-dependent problems including non-linear material properties and different physical aspects are examined. Afterwards, advanced post-processing techniques enable expressive evaluations and visualizations of computed results.

A classical approach for the solution of the aforementioned problems is to treat them monolithically as a single complex problem. All physical aspects are summarized into one equation system and one numerical method and one formulation is chosen, which solves the total problem. The post-processing is executed using the same model along with the same numerical method as for the solution of the equation system. An advantage of such an approach is that the numerical model is very precise and clear. On the other hand, the properties of the chosen numerical method must fit to all steps of the simulation process. Hence, the choice of an appropriate numerical method is a compromise between general 
applicability and efficiency. Furthermore, this choice is a challenging task, especially for users of numerical methods.

A decomposition of a single field problem into several sub-problems is a very promising approach to tackle complex problems. Numerical methods can be chosen separately for the solution of each sub-problem and for its post-processing. That means, properties of numerical methods fit much better to the demands of partial simulation steps than in the case of only one method for the total problem. On the other hand, data must be prepared for an exchange between different sub-problems.

Here, a very flexible and powerful concept is presented, which combines advantages of different numerical methods and formulations for the solution of complex or coupled field problems. The focus is on boundary element methods including various formulations, compression techniques, and post-processing approaches. Next, several mixtures of boundary element methods with other numerical methods are discussed. Classical coupling of numerical methods is considered as well as modern techniques like software agents.

\section{BOUNDARY ELEMENT METHODS}

The main idea of the boundary element method (BEM) is to describe a field problem only based on values on domain boundaries (Brebbia et al. [1]). Hence, a modelling and discretization of surfaces suffices to completely define the considered problem. Therefore, BEM is a very attractive method for science and engineering applications (Buchau [2]). Especially open boundary problems or problems with large distances between objects are solved very efficiently and with high accuracy.

\subsection{BEM formulations}

A concrete BEM formulation depends on the specific physical problem, which is solved. Furthermore, several formulations are possible for the same problem. For instance, a static electric field $\boldsymbol{E}$ can be described completely using a scalar electric potential $u$ with

$$
\boldsymbol{E}=-\nabla u .
$$

Based on Green's theorem the potential $u$ in a point $r$ within a closed domain $\Omega$ is obtained from the value of $u$ at the domain boundary $\partial \Omega$ and its normal derivative $\frac{\partial u}{\partial n^{\prime}}$ by

$$
u(\boldsymbol{r})=\int_{\partial \Omega} G\left(\boldsymbol{r}, \boldsymbol{r}^{\prime}\right) \frac{\partial u\left(\boldsymbol{r}^{\prime}\right)}{\partial n^{\prime}} \mathrm{d} A^{\prime}-\int_{\partial \Omega} \frac{\partial G\left(\boldsymbol{r}, \boldsymbol{r}^{\prime}\right)}{\partial n^{\prime}} u\left(\boldsymbol{r}^{\prime}\right) \mathrm{d} A^{\prime}
$$

with Green's function of three-dimensional Laplace equation

$$
G\left(\boldsymbol{r}, \boldsymbol{r}^{\prime}\right)=\frac{1}{4 \pi} \frac{1}{\left|\boldsymbol{r}-\boldsymbol{r}^{\prime}\right|}
$$

Equation (2) is only applicable, if objects with a closed surface and a defined volume are considered. In the case of very thin objects, it would be necessary to add artificial boundaries to close all surfaces. Then, another BEM formulation

$$
u(\boldsymbol{r})=\frac{1}{\epsilon_{0}} \int_{A} \sigma\left(\boldsymbol{r}^{\prime}\right) G\left(\boldsymbol{r}, \boldsymbol{r}^{\prime}\right) \mathrm{d} A^{\prime}
$$


based on surface charge densities $\sigma$ is more suited. The free space permittivity is $\epsilon_{0}$ and $A$ are the surfaces of closed or thin objects.

An important distinction between eqn (2) and eqn (4) is the scope where the equations can be applied. Equation (2) is valid only inside a closed domain. In the case of multiple domains, several sets of boundary values are required to describe the field for each domain. In contrast, eqn (4) is valid in total space and no distinction between domains is necessary even in the case of multiple closed domains.

In practice, both BEM formulations are applied for the solution of static electric field problems. The direct formulation using eqn (2) is for instance very advantages for problems with layered objects, since then the matrix of the corresponding linear system of equations is relatively sparse. The indirect formulation based on eqn (4) is well suited for thin structures. In total, there are pros and cons for both BEM formulations and it depends on the concrete example in which formulation is more precise and more efficient.

However, the computation of the solution of a problem is an important step but only a first step in numerical methods. Evaluation and interpretation of results is mandatory in engineering applications.

A common approach is to use the same BEM formulation for both, the computation of the solution of problem and the post-processing. Then, computed values on boundaries can be used directly for post-processing. Furthermore, it is necessary to store only formerly unknown values and to integrate known boundary values.

That means in the example of a static electric field that the potential is given at the electrodes and it is not necessary to store potential values in the element nodes of electrodes. Only its normal derivative is computed and stored for each element node. During the postprocessing, eqn (2) is evaluated using the given boundary values and using the computed missing boundary values.

An advantage of such a self-evident approach is that the amount of required main memory is minimized and no unnecessary computations are executed. A significant drawback is that the approach is very restricted. All details of the formulation, which are necessary for the solution of the problem, must be considered and implemented for the post-processing, too.

Nowadays, enough main memory is available for most applications. Hence, a more flexible approach, which consumes a little more memory, is recommended. Here, the solution of the problem and the post-processing are consequently separated. That means the solution of the problem and its post-processing are computed independently of each other.

The solution of the problem is calculated using a BEM formulation, which is predestined for a stable and efficient solution of the problem. All computational steps are optimized for the solution process. Then, the solution is converted into boundary values for the postprocessing. These values contain both original boundary values and computed values on the boundaries.

The post-processing step is significantly simplified, if all given and computed boundary values are treated in the same manner. In case of a direct formulation like eqn (2) is applied, potential and its normal derivative are stored for each element node. Then, no distinction is necessary for the evaluation of eqn (2) during the post-processing. Furthermore, the BEM formulation can be changed for the post-processing. For instance, the solution of the problem can be obtained using a direct BEM formulation and the post-processing can be done based on an indirect formulation and vice versa.

Another benefit of that separation is that different BEM implementations can be used. The solution is computed and stored in a general applicable format independently of the 
formulation and problem description. Another process imports the boundary element mesh along with all nodal values. That enables flexible field computations and impressive visualization techniques for arbitrary BEM problems (Buchau \& Rucker [3]).

\subsection{Matrix compression techniques}

Since the matrix of the linear system of equations is dense or block-wise dense, a matrix compression technique is required to solve complex field problems. In the last decades, several matrix compression techniques have been developed. They exploit the dependency of Green's function on the distance between source point and evaluation point and approximate Green's function in the case of far-field interactions.

One class of matrix approximation techniques is based on series expansions of Green's function along with hierarchical grouping schemes for the boundary elements. The most popular representative of this class is the fast multipole method (FMM) (Greengard \& Rokhlin [4]). The series expansion depends on the used Green's function. Hence, each kind of problem or basic equation, respectively, requires that series expansion in spherical coordinates for three-dimensional problems. Furthermore, transformations of these series expansions must be available, which convert the coefficients of the series expansion from one local coordinate system into coefficients of the series expansion of another local coordinate system. Unfortunately, the computation of these transformations is relatively expansive. On the other hand, the FMM is a very flexible tool both for the solution of the problem and for the post-processing, since it depends only on geometrical position of boundary elements and evaluation points (Buchau et al. [5]). In both cases, very high compression rates are obtained and very large problems can be solved efficiently.

Another class of compression techniques is based on direct matrix operations. This is used for instance for the adaptive cross approximation technique (ACA) (Bebendorf [6]). The ACA applies a hierarchical matrix clustering based on the distance between source point and evaluation point. Thus, an excellent numbering of element nodes is required to achieve high compression rates. Each matrix block is then approximated by a few rows and columns. Since the computed rows and columns correspond to those of the original dense matrix, the computation of matrix elements is the same for the ACA as for the classical BEM. Therefore, the ACA is applicable to general BEM formulations. Some modifications of matrix assembly are necessary to avoid multiple expensive computations of integrals over the same boundary element. The ACA is very advantageous for the solution of the linear system of equations with a large number of iterations, for instance, in combination with a time-dependent or non-linear solver, because matrix by vector products are evaluated very fast. On the other hand, post-processing based on ACA is limited to regular grids of evaluation points but a flexible post-processing is not possible.

In total, matrix compression techniques are very attractive for BEM computations, since both the computational costs and memory requirements are significantly reduced (Buchau et al. [7]). The choice of an appropriate matrix compression technique depends on the concrete problem and BEM formulation as well as on the desired post-processing. If the same matrix compression technique is applied both to the solution of the problem and the post-processing, an optimal method is difficult to find. Here, a mixture of matrix compression techniques is recommended. A separated treatment of solution step and post-processing step as discussed in the previous section enables an independent choice of matrix compression technique, too. Furthermore, it is possible to choose different algorithms or implementations for the same matrix compression method. 
The structure of the matrix of the linear system of equations is predefined by the given boundary element mesh. Hence, a matrix compression method can be highly optimized and parallelized for that case. An important property of a fixed boundary element mesh is that clustering and grouping for ACA or FMM is fixed, too. Both matrix block of ACA and octree structure along with transformation coefficients of the FMM can be precomputed and reused in each iteration step of an iterative solver of the linear system of equations (Axelsson [8]).

If not a fixed grid is used for the post-processing but a flexible meshfree post-processing is applied (Buchau \& Rucker [3]), adapted versions of matrix compression techniques are more efficient (Buchau \& Rucker [9]). There, the position of evaluation points is determined during the calculation of visualization objects like field lines. Hence, evaluation points are added to the hierarchical octree based grouping scheme of FMM during the post-processing. This requires a flexible implementation of both the octree and the FMM algorithm. Furthermore, a reversed FMM algorithm reduces the number of FMM operations significantly. A drawback is that optimization and parallelization is more difficult.

As demonstrated in that example, even the same matrix compression technique should be used in very different manners for the solution of the problem and for the post-processing. Hence, different implementations are often advisable. Then, all aspects of performance and flexibility can be considered appropriately.

\subsection{Post-processing}

Post-processing BEM operations differ significantly from BEM operations for the solution of the problem, not only for the matrix compression technique as shown in the previous section. A second important topic is BEM integrals, especially singular or nearly singular integrals.

In many cases, the number of considered boundary element types is limited. Hence, a small number of singular or nearly singular integral types have to be implemented. Then, these integrals are normally highly optimized to shorten CPU time.

In the case of post-processing, different integral kernels are used. The reason is that not only the field value, which is the solution of the problem, but also derivatives of that field value are evaluated. Of course, an optimized implementation with low computational costs is favoured but flexibility has higher priority in that case. Singular, nearly singular, nearly strong singular or nearly hyper-singular integrals can be implemented in a relatively general way. For instance, sub-division of boundary elements for an application of local polar coordinates can be generalized. Then, special treatment of these integrals is shifted into weights of integration points in the implementation. The overhead for some additional multiplications, which can be summarized in an optimized implementation, is relatively small but a huge number of element types, formulations, and field values can be taken into account.

Another advantage of a separated post-processing is that different techniques can be applied. A classical approach is to create a grid or auxiliary volume mesh and to compute field values in that mesh nodes. Then, post-processing tools for FEM are applicable. The main drawback of such an approach is that field values are computed in points, which are not necessary for visualization. On the other hand, implementation is relatively easy and already existing code can be reused.

If post-processing is separated from the solution of the problem, only boundary values of for instance the potential are transferred to the post-processing tool. The evaluation of these 
values can be done with an arbitrary BEM formulation, an arbitrary compression technique and an arbitrary implementation of BEM integrals. The post-processing computations can be integrated into the visualization tool or visualization objects are computed separately and then transferred to the visualization tool (Buchau \& Rucker [3]).

For instance, basic concepts of FMM and isovalue search are common (Wilhelms \& Gelder [10], Buchau \& Rucker [11]). An integration of BEM with FMM into an algorithm for computation of isosurfaces offers the opportunity to interweave both. The same octree is then used to group boundary elements and evaluation points for a reversed FMM algorithm and FMM series expansions are applied to interpolate field values for an isovalue search method with high accuracy.

Furthermore, an integration of BEM operations directly into the post-processing computations enable a much better control of accuracy of computed visualization objects. Adaptive integration methods like a Runge-Kutta-Fehlberg method adjust step size automatically for precise field line computations (Fehlberg [12]). However, a fixed grid of evaluation points would limit the total accuracy by rough interpolations between the evaluation points but a direct evaluation of BEM integrals improves accuracy significantly although the computational costs are kept low.

\section{COUPLED NUMERICAL METHODS}

Boundary element methods are applicable to a wide range of problems. However, non-linear or inhomogeneous media cannot be integrated into BEM easily. In that case, the finite element method (FEM) is very attractive. A classical approach is to couple BEM and FEM by integrating both methods into one monolithic equation system. Another approach is to extend a coupling of BEM with other methods within the meaning of the concept presented in the previous section.

Although FEM is well suited to consider nearly arbitrary material properties, modelling of large surrounding air domains is very expensive. Boundary layers modelled with infinite elements represent alternatives, but unfortunately field values must also be computed precisely in air domains in many engineering applications. A very interesting approach is to apply FEM for the solution of the problem and BEM for the post-processing. Then, the FEM model is acceptable small and the surrounding domain is discretized as small as possible. Of course, field values in the surrounding domain are too inaccurate but field values at the surfaces of examined objects are accurate enough. These values are used for a BEM post-processing.

A typical situation for that approach is the computation of the radiated electromagnetic field of a smartphone antenna. The structure of a modern smartphone is very complex especially the antenna system. Hence, it is advisable to model the device using FEM. Then, a very small air domain along with absorbing boundary conditions suffices to compute the electromagnetic field of the device with high accuracy. Unfortunately, the radiated electromagnetic field in the air domain is of large interest for the design of a smartphone. However, a small air domain avoids visualization of the radiation pattern in the far-field. Here, a BEM post-processing is a promising alternative method. The complex structure of the device does not matter, since only boundary values on the device surface are needed for BEM. The infinite surrounding air domain is very precisely considered by Green's function. Especially a meshfree BEM post-processing results in accurate and expressive visualizations of the radiated electromagnetic field. 
A combination of BEM with other methods like FEM for general problems or physical optics or universal theory of diffraction for electromagnetic wave problems is very attractive for the computation of the solution of the problem. Instead of a classical coupling of these methods, an application of software agent systems offers completely new possibilities (Jüttner et al. [13]). A complex problem is subdivided into smaller problems similar to domain decomposition methods. Each sub-problem is solved by a software agent, whose aim is to solve its local sub-problem most efficiently. The advantage of that approach is that arbitrary numerical methods along with their implementation can be used without modification of the corresponding code. Only an interface for data exchange and its coordinate based evaluation is required, which enables the independent selection of an appropriate numerical method for each sub-problem. Thus, boundary or domain values are computed and exchanged with software agents of all other sub-problems. Software agents not only coordinate the solution process on their own, but they also have the ability of choosing a numerical method. Hence, this approach is very attractive for a combination of several well-developed software packages with different numerical methods. Same holds for the visualisation of simulation results. Since software agents solve only sub-problems, the post-processing can benefit from the separated results by processing data only within the region interest.

The concept of coupling of different numerical methods for the solution of a complex problem can be extended to the solution of multiphysics field problems (Jüttner et al. [14]). There, different physical aspects of a complex problem are assigned to software agents, which are on the top layer of the solution process. Each physical sub-problem is handled by a software agent, which chooses the numerical method for the solution of the sub-problem. Based on performance criteria rated by a user this results in a mixture of different numerical methods within a solution sequence. Furthermore, also different numerical libraries and solver configurations can compete within a calculation process to provide the result as fast as possible (Jüttner et al. [15]). Alternatively, also the calculation of parameter sweeps and optimisation tasks benefit from this naturally parallel setup.

A significant advantage of solving a multiphysics field problem with the help of software agents is that not only numerical methods are chosen for each physical problem but also different meshes can be used. Examples are overlapping domains within a multiphysics problem where the physics suggest different meshes based on the considered different material properties. The values, which are needed for another physical sub-problem, are computed in the same manner as the aforementioned post-processing approach. Furthermore, a software agent is able to fetch values from other agents, which compute these values on demand.

For some multiphysics problems the coupling is relatively weak. For instance, if the temperature distribution inside an electric conductor is approximately homogeneous, electric conductivity is homogeneous, too. Then, a BEM can be used for the solution of the sub-problem. If the temperature distribution is inhomogeneous along with inhomogeneous electric conductivity, a FEM is preferred. This change of the numerical method easily realized by the software agent system. This also includes iterative domain decomposition approaches based on the Schwarz formulation. Sub-problems with a strong coupling benefit from the redundancy and the fault tolerance during the calculation when using a segregated and distributed approach.

\section{NUMERICAL RESULTS}

Two examples are considered to demonstrate that the presented concepts work reliable and advantageous in practice. 


\subsection{High voltage insulator}

The electric field of a high voltage insulator is studied as a first example. Since the insulator has linear material properties, an indirect BEM formulation has been chosen to compute the electric field strength between the electrodes inside the insulator and in the surrounding air domain.

The surfaces of the device have been discretized using second order quadrilateral boundary elements. A Galerkin method is applied to obtain a linear system of equations, which is solved iteratively using GMRES with a Jacobi preconditioner. The matrix of the equation system is compressed with the help of a classical FMM. Singular integrals are computed using highly optimized code. All methods for the solution of the problem are implemented in $\mathrm{C}++$ and parallelized with OpenMP standard.

The results are extracted for a post-processing based on another BEM implementation. There, the focus was on flexibility and not on maximum performance. The post-processing software is implemented in C\# and has interfaces to the above-mentioned BEM solver and to commercial FEM code. Meshes are imported too. Since the post-processing is independent of the solution, order of elements or type of elements can be changed. Especially linear triangles are often used in visualization because of hardware supported rendering capabilities. Singular integrals are implemented in a very flexible manner to enable the treatment of all kind of boundary elements. Visualization algorithms are integrated to apply a powerful meshfree computation of field lines (Fig. 1) or isosurfaces (Fig. 2).

\subsection{Wet casing of an antenna including a lens}

Now, the propagated wave of an antenna within its wet casing with an inserted lens is evaluated. Therefore, a FEM model of the electric field as dependent variable is created and

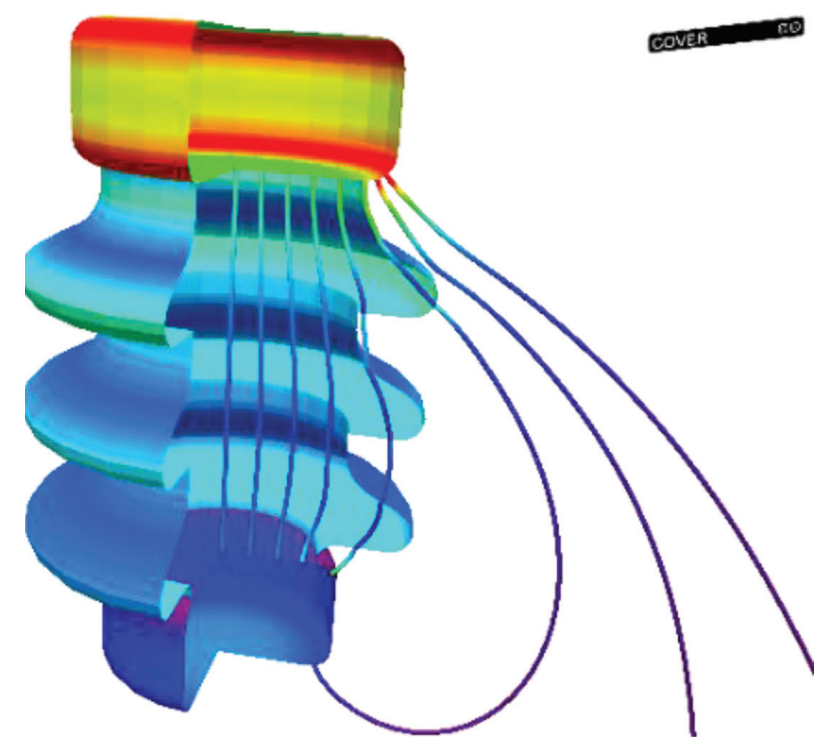

Figure 1: Field lines of the electric field strength of a high voltage insulator. 


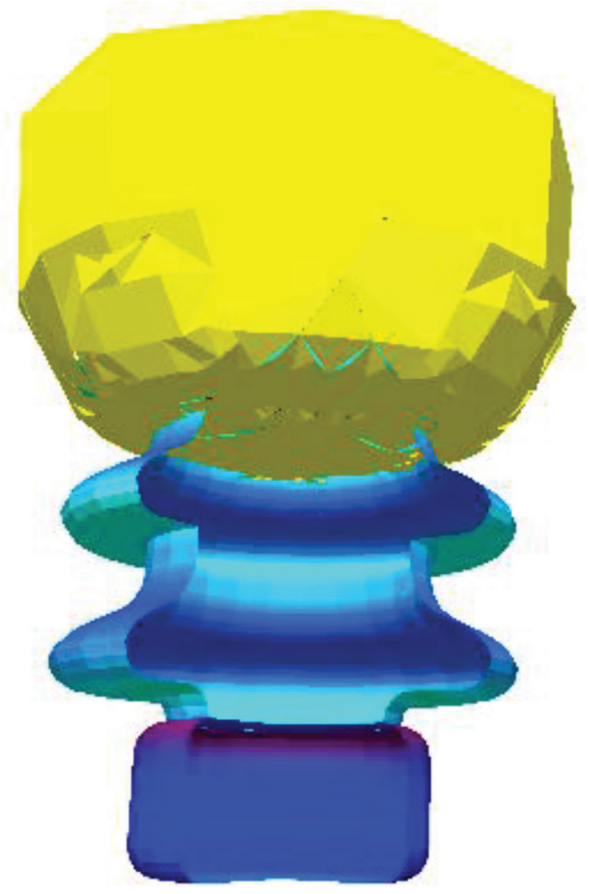

Figure 2: Isosurface of the electric potential of a high voltage insulator.

updated in a next engineering step by an additional description of the temperature. This leads to a bi-directionally coupling between the dependent variables and enables the regard of electric losses within the materials and their temperature dependent behaviour within the simulation.

The applied software agent system performs a physics based decomposition of the problem. Since the initial model only contains a description of the electric field, redundant resources are used automatically to evaluate a best performing solver. The proposed solver is used after updating the model. The physics based decomposition now generated sub-problems that use the already calculated results as initial values for the consequently following iterative calculation process. This decomposition further allows the separated treatment of the mixed complex and real degrees of freedom by using optimised numerical libraries. A sub-problem dependent application of the methods BEM and FEM for different domains is also possible (Jüttner et al. [15]).

The overall result of the calculation process is shown in Fig. 3. For its calculation, different discretisations for the sub-problems were used. Different shape functions were used by the software agents due to the considered physics. Distinct discretisation were chosen since the material properties within the physics differ.

\section{CONCLUSIONS}

A concept for an application of different numerical methods for the solution of complex field problems has been shown. Especially boundary element methods are predestined for such a coupling of numerical methods. A separation of solution of the problem and post-processing 


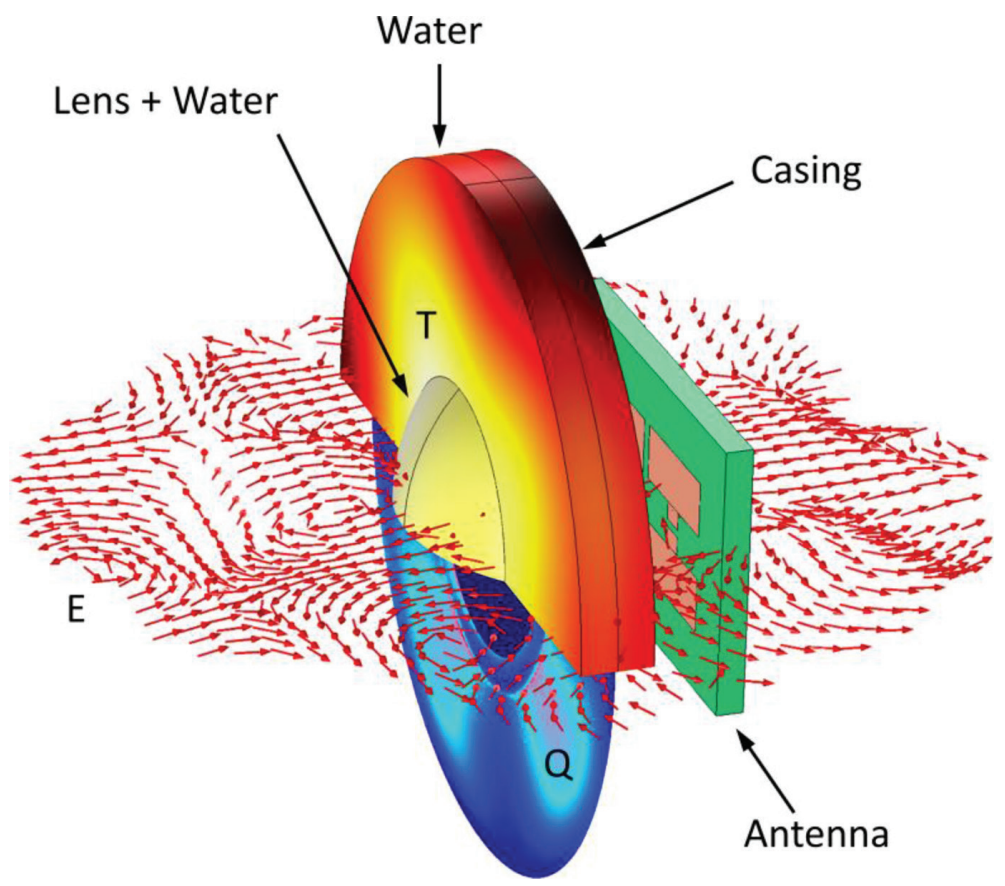

Figure 3: Multiphysics result for a heated lens within its casing by a patch antenna array.

enables rich possibilities for efficient and flexible computations. Different algorithms and implementations of the same method or different methods can be combined in nearly arbitrary manner. Modern software techniques like software agents support this concept and extend it to the solution of complex multiphysics field problems.

\section{REFERENCES}

[1] Brebbia, C.A., Telles, J.C.F. \& Wrobel, L.C., (eds.), Boundary Element Techniques, Springer-Verlag: Berlin and New York, 1984.

[2] Buchau, A., Application of Fast Boundary Element Methods in Engineering and Science. Proceedings of ICCES 2017 International Conference on Computational \& Experimental Engineering and Science, Funchal, 2017.

[3] Buchau, A. \& Rucker, W.M., Feasibility of a meshfree post-processing for boundary element methods. Boundary Elements and Other Mesh Reduction Methods XXXVIII, pp. 327-338, 2015.

https://doi.org/10.2495/bem380261

[4] Greengard, L. \& Rokhlin, V., A New Version of the Fast Multipole Method for the Laplace Equation in Three Dimensions. Acta Numerica, pp. 229-269, 1997.

[5] Buchau, A., Rieger, W. \& Rucker, W.M., Fast field computations with the fast multipole method. COMPEL, 20(2), pp. 547-561, 2001. https://doi.org/10.1108/03321640110383861

[6] Bebendorf, M., Approximation of boundary element matrices. Numerische Mathematik, 86(4), pp. 565-589, 2000.

https://doi.org/10.1007/pl00005410 
[7] Buchau, A., Rucker, W.M., Rain, O., Rischmüller, V., Kurz, S. \& Rjasanow, S., Comparison between different approaches for fast and efficient 3D BEM computations. IEEE Transactions on Magnetics, 39(3), pp. 1107-1110, 2003. https://doi.org/10.1109/tmag.2003.810167

[8] Axelsson, O., Iterative Solution Methods, Cambridge University Press, Cambridge, UK, 1996

[9] Buchau, A. \& Rucker, W.M., Meshfree computation of field lines across multiple domains using fast boundary element methods. IEEE Transactions on Magnetics, 51(3), pp. 1-4, 2015. https://doi.org/10.1109/tmag.2014.2359520

[10] Wilhelms, J. \& van Gelder, A., Octrees for faster isosurface generation. ACM Transactions on Graphics, 11(3), pp. 201-227, 1992.

https://doi.org/10.1145/130881.130882

[11] Buchau, A. \& Rucker, W.M., A meshfree isosurface computation method for boundary element methods. International Journal of Computational Methods and Experimental Measurements, 5(5), pp. 647-658, 2017.

[12] Fehlberg, E., Klassische Runge-Kutta-Formeln fünfter und siebenter Ordnung mit Schrittweiten-Kontrolle. Computing, 4(2), pp. 93-106, 1969. https://doi.org/10.1007/bf02234758

[13] Jüttner, M., Buchau, A., Rauscher, M., Rucker, W.M. \& Göhner, P., Software Agent Based Domain Decomposition Method, 15th International IGTE Symposium, Graz, Austria, pp. 89-94, 2012.

[14] Jüttner, M., Buchau, A., Vögeli, D., Rucker, W.M. \& Göhner, P., Iterative Software Agent Based Solution of Multiphysics Problems, Scientific Computing in Electrical Engineering, Springer, pp. 123-131, 2016.

https://doi.org/10.1007/978-3-319-30399-4_13

[15] Jüttner, M., Grabmaier, S., Vögeli, D., Rucker, W.M. \& Göhner, P., Coupled multiphysics problems as market place for competing autonomous software agents. IEEE Transactions on Magnetics, 53(6), pp. 1-4, 2017.

https://doi.org/10.1109/tmag.2017.2659228 